\title{
A Simple Accommodation for Ulnar Nerve Paraesthesia Following Body Impacts from Mechanical Energies
}

\section{Michael A Persinger ${ }^{*}$ and Linda S St-Pierre}

Clinical Neuroscience Laboratory, Behavioural Neuroscience, Biomolecular Sciences and Human Studies Programs, Laurentian University, Sudbury, Ontario P3E 2C6, Canada

*Corresponding author: Michael A Persinger, Clinical Neuroscience Laboratory, Behavioural Neuroscience, Biomolecular Sciences and Human Studies Programs, Laurentian University, Sudbury, Ontario P3E 2C6 Canada, Tel: 01-705-675-4824; E-mail: mpersinger@laurentian.ca

Rec date: May 30, 2016; Acc date: Jul 22, 2016; Pub date: Jul 24, 2016

Copyright: (c) 2016 Persinger MA and St-Pierre LS. This is an open-access article distributed under the terms of the Creative Commons Attribution License, which permits unrestricted use, distribution, and reproduction in any medium, provided the original author and source are credited.

\begin{abstract}
Acquired painful or distracting paraesthesia of the lateral two fingers subsequent to a strong mechanical impact to the body can also be elicited clinically by point stimulation around the ipsilateral periscapular region. This neurological (ulnar) demonstration offers an alternative attribution of the etiology for the patient. A simple mechanical procedure that can be self-administered by the patient over several weeks minimizes the symptom and can facilitate sense of control of the unwanted experiences.
\end{abstract}

\section{Opinion}

We have assessed hundreds of patients over the last four decades that had sustained significant impacts of mechanical energies to their brains and bodies [1]. Most occur as a result of motor vehicle incidents. A relatively frequent complaint (about one third of the population) that often dominates the subjective presentation of the patient is the experience of paraesthesia (unpleasant "tingling", odd "electrical" sensations) which is typically isolated to the lateral two fingers that correspond to the distribution of the ulnar nerves. Reports of these experiences for the left hand are more frequent (about $80 \%$ of the cases) than the right.

The paraesthesia can be easily elicited by the clinician when mild to moderate pressure is applied with the fingers along and within the boundary of the ipsilateral scapula. The patient identifies these experiences as similar to those unpleasant sensations that have occurred persistently and spontaneously since the injury.

Although many of the post-impact injuries to both soft tissues and the cerebrum cannot be easily accommodated by patient-generated interventions, we have found a technique that when employed appropriately will eliminate or minimize the occurrence of the unpleasant paraesthesia. The procedure was discerned while exploring various self-applicable, mechanical methods to relax apposing muscle groups. As shown in Figure 1, the patient is instructed to articulate each arm to reach and to intercalate with the fingers from the other hand for about $30 \mathrm{~s}$ two or three times each day until the patient achieves the position indicated or the symptoms are maximally reduced.

Symmetry (left hand over the shoulder, right hand under the back and vice-versa) is emphasized. They are instructed to not over-strain the stretch but to work towards this capacity. In all of the patients we have seen who reported a troubling ulnar paraesthesia the closest distances (without distress) at the beginning between the tips of the fingers of each hand were separated by $15 \mathrm{~cm}$ to more than $30 \mathrm{~cm}$. Of the approximately 60 patients for whom we have second assessment (about a year later) data more than $90 \%$ reported conspicuous benefits from this procedure.
Careful inspection of the skin and muscular as well as preliminary electromotor measurements have revealed the expected discrepancy between flexor and extensor functions as well as subtle hypertrophy that we attribute to edema within the periscapular musculature. We suspect but cannot prove that the correlative pressure on the peripheral components of ulnar nerve is the source of the paraesthesia. The resultant self-control also contributes to the patient's positive perception of the saliency of the neurological examination.

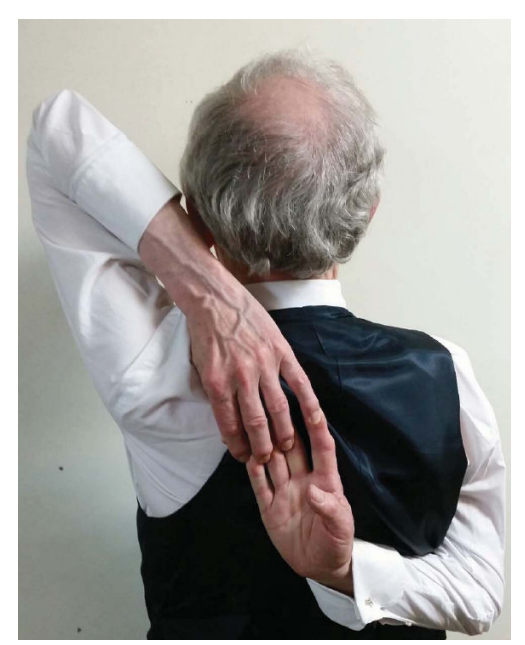

Figure 1: Demonstration of the ultimate level of flexor-extensor balance that minimizes ulnar paraesthesia from anomalies within the scapular region. When most patients begin they can barely flex either arm. Brief daily, self-initiated gradual stretching may take several weeks to achieve criteria (Thanks to Professor Nicolas Rouleau for taking the picture). 
Citation: Persinger MA, Pierre LS (2016) A Simple Accommodation for Ulnar Nerve Paraesthesia Following Body Impacts from Mechanical Energies. J Neurol Disord 4: 280. doi:10.4172/2329-6895.1000280

Page 2 of 2

\section{References}

1. Tiller S, St-Pierre LS, Persinger MA (2013) Absence of quantitative improvement in neuropsychological profiles in patients who exhibit moderate brain impairment: Comparisons of cross-sectional and longitudinal data (1 to 4 years post injury). J Behav Brain Sci 3: 225-228. 